\title{
Increasing ECE Student Excitement through an International Marine Robotics Competition
}

\author{
Major Joseph Lee Heyman, United States Military Academy
}

Joseph L. Heyman is an Instructor in the Department of Electrical Engineering and Computer Science, United States Military Academy. He holds a B.Sc. degree in Electrical Engineering from the United States Military Academy and an M.Sc. in Electrical and Computer Engineering from Carnegie Mellon University.

\section{Dr. Wenli Huang, U.S. Military Academy}

Wenli Huang is a Professor of Electrical Engineering at the Department of Electrical Engineering and Computer Science at the U.S. Military Academy, West Point, New York. She received her Ph.D in electrical engineering from the University of Connecticut in 1995. She is currently a research member of the Photonics Research Center at West Point. Her research interests include halftone image processing and design/modeling of nanostructure optoelectronic devices. She is a Senior Member of IEEE and a member of Phi Kappa Phi and Eta Kappa Nu honor societies.

\section{Prof. Guangming Xie, Peking University}

Guangming Xie received his B.S. degrees in Applied Mathematics and Electronics \& Computer Technology, his M.E. degree in Control Theory and Control Engineering, and his Ph.D degree in Control Theory and Control Engineering from Tsinghua University, Beijing, China in 1996, 1998 and 2001, respectively. Then he worked as a postdoctoral research fellow in the Center for Systems and Control, Department of Mechanics and engineering Science, Peking University, Beijing, China from July 2001 to June 2003. In July 2003, he joined the Center as a lecturer. Now he is an Associate Professor of Dynamics and Control. He is also a Guest Professor of East China Jiaotong University. He is an associate editor of the International Journal of Advanced Robotic Systems and the Journal of Information and Systems Science. His research interests include smart swarms, hybrid and switched systems, complex networked control systems, multi-agent systems, multi-robot systems, and biomimetic robotics.

\section{Pongpat Taephanitcharoen, United States Military Academy}

Pongpat Taephanitcharoen is an exchange cadet from Thailand who is currently pursuing a B.Sc. in Electrical Engineering at the United States Military Academy, West Point, NY. 


\section{Increasing ECE Student Excitement through an International Marine Robotics Competition}

\section{Introduction}

In this paper we describe our experience with the 1st International Underwater Robot Open Competition and the Advanced Individual Studies course that we created to support our students' participation in the competition. We begin by briefly describing the background and history of the competition. We then discuss the prior collaboration between the United States Military Academy (USMA) and Peking University that led to USMA's participation in the competition. Within this discussion of the collaboration, we also include representative funding and budget considerations. Understanding of the Advanced Individual Studies course created to support the competition would not be complete without an introduction to the robotic platform involved. Therefore, that section is presented next. Following this discussion, we present a general description of Advanced Individual Studies in Electrical Engineering (EE489) at USMA and then discuss the specific EE489 course created to support participation in the competition. We then describe our students' experiences during the trip to a foreign country and during the competition itself. Finally, we present our conclusions and lessons learned during the process.

\section{Competition Background.}

The concept of the Robotic Water Polo (RWP) competition that eventually became the Underwater Robot Competition of China was first proposed in $2007^{1,2}$. The stated goal of the competition is to spur the interest of undergraduate students in studying marine robotics and to stimulate research in both academics and industry. Early prototypes of RWB featured a competition between two teams of two to three underwater robots ${ }^{1}$. Since these underwater robots are biologically inspired, they are commonly referred to as robotic fish. Students program the robotic fish with various control strategies to compete in water polo matches. Teams can score points, or goals, by pushing an inflatable ball into the opposing team’s goal.

The first RWP competition was held in 2008. Since then, the competition has expanded to include more than just robotic water polo. It now includes four leagues: global-vision, 2D simulation, autonomous, and free innovation ${ }^{3 .}$ Robotic water polo (1vs1 and 2vs2) is now one of the six total games in the global-vision league. The other four games in the global-vision league feature a single team attempting to complete a task using either one or two robotic fish. These four games are: relay race with ball, avoiding barriers with ball, cycling with ball, and ballpassing relay ${ }^{4}$ Each team is scored based on the time taken to accomplish the specific task. The team with the fastest time is declared the winner of the game.

In 2012, our team only competed in the global-vision league. Most discussion in this paper will be limited to that league and the platform used is further described in Section 4. However, here we offer a short description of the other 3 leagues. In the autonomous league, all sensing and control must be implemented on the robot. In addition, this league allows for robots that aren't biometrically inspired. This is in contrast to the global-vision league, which includes an external camera-based vision system, external computer control, and the requirement that the robots are 
biologically based. The 2D simulation league is based on graphical simulation of the dynamics of the robotic fish used in the global-vision league. This league includes simulated 1vs1 and 2vs2 water polo and many other games that have more "fish" than the physical based leagues. The free innovation league is open to any robot, algorithm, or software loosely based on any of the other leagues. Some examples from 2012 included a robotic dolphin, a robotic Chinese dragon, and a wolf-pack hunting algorithm implemented with the robotic fish.

With the expansion to more leagues and the inclusion of international teams, the competition was renamed the Underwater Robot Competition of China / International Underwater Robot Open Competition. Over 40 different schools have competed in these yearly competitions. The 2012 version of the competition included over 120 teams from China, the Netherlands, South Korea, and the United States ${ }^{3}$.

\section{Collaboration Background.}

One of USMA's many visions is to prepare graduates for a changing world. To support an institutional goal of developing graduates who appreciate both the diversity in culture and the challenges of performing duties in a multicultural environment, our department arranged to send a few students for an Individual Advanced Development (IAD) program to China. The program takes place during the summer break and lasts for about two weeks. The primary purpose of the program is for our students to conduct collaborative engineering projects together with the students at Chinese universities. We hope, through living in China and interacting with Chinese students, our students will be immersed in Chinese culture; gain regional experiences, and perform in a multi-lingual environment. The students are chosen from majors in electrical engineering, computer science, or information technology and they have had at least one year of Chinese language instruction. The average cost for the trip is about $\$ 3500.00$ per student for a two week trip. This trip is longer than necessary for competing in the competition, but gives students more time to explore a foreign culture. Costs could be slightly reduced by shortening the length to cover only the two days of the competition. In the case of USMA, the trip is fully sponsored by our international office at no cost to the students or academic department.

In 2011, one very interesting collaborative opportunity our students came across was with the Intelligent Control Laboratory at Peking University. Several Chinese graduate students had been working on building and controlling robotic fish. Our students were very excited about the project and had a great deal of discussions with the Chinese students about the mechanics, controls, communications, and image processing involved in the project. We learned that the underwater robot platform developed at Peking University has been used for robotic fish competitions annually. Our students were very enthusiastic about taking part in such a competition. The contest not only serves the aforementioned goal of cultural exposure but is also a great way to motivate our students to study robotics throughout the year. Peking University cordially invited our students to participate in the competition. Moreover, Peking University has generously provided us with the platform including all the necessary equipment and software for our students to study and practice. The system was sold for as much as $\$ 30,000$ to some universities. The overall description of the platform is outlined in the next section. 


\section{The Competition Platform.}

The underwater robot platform developed at the Peking University consists of four subsystems ${ }^{1,2}$ : the robotic fish, image capturing and processing, decision making and wireless communication. The layout of the platform is shown in Figure 1. The pool dimensions are rectangular, 3 meters by 2 meters, with a water depth of .5 meters. The information about the robotic fish and their surroundings is captured by an overhead camera. The camera interfaces with a computer to serve together as the vision system and effectively collect real time data for the positions and directions of the fish as well as the surrounding objects, such as water polo ball and obstacles. This data is then sent to the decision making subsystem. Then, based on this data and specific control strategies for different tasks, the decision making subsystem produces corresponding control commands and transmits them to every robotic fish through the wireless communication subsystem.



Figure 1: Hardware Layout of Robotic Fish Water Polo Platform ${ }^{1}$

Figure 2 shows a picture of the robotic fish. The robotic fish is divided into three main components: the head, the body, and the tail. The head consists of a control circuit board, a communication module, a rechargeable battery and a power switch. The body is used to move the fish and consists of three DC servo-motor powered inter-connecting joints in order to simulate the oscillating motion of a natural fish. Lastly, the tail provides stability and adds to the aesthetic image of the robotic fish ${ }^{1,2}$. The tail also helps the user to differentiate among multiple fish, as they can easily be color marked for distinction. 


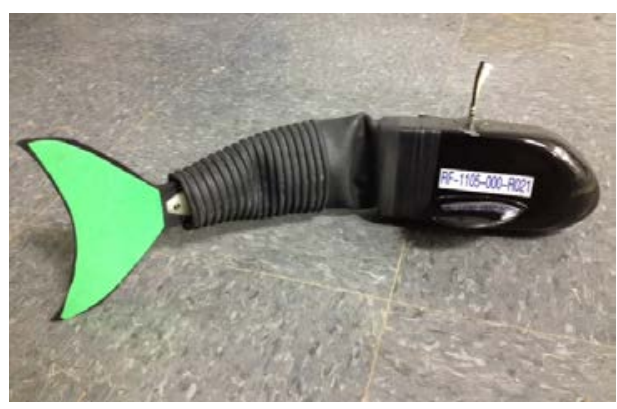

Figure 2: Physical Structure of Robotic fish

The center piece of the platform is the decision making subsystem. Developed by Peking University, the decision making subsystem is centered on a graphical user interface (GUI), which conveniently gathers necessary information, allows the user to choose different robotic fish strategies, and set parameters of the environment (goals, obstacles, etc.) with ease. The software platform is established utilizing Visual C++ Service Pack 5 on Windows environments. The GUI maps the robotic fish strategies to a C-file in the directory, which can then be changed by the user. Figure 3 shows the main window of the GUI.

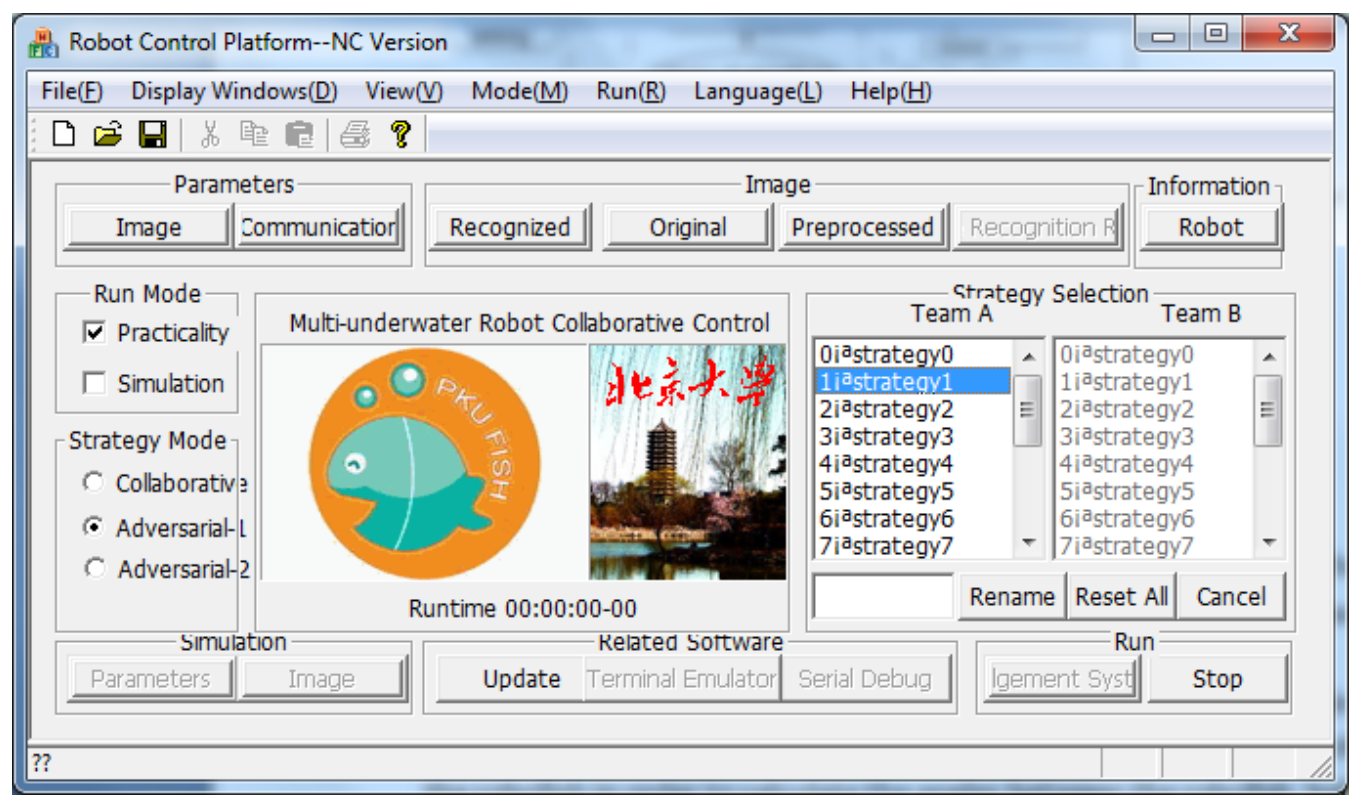

Figure 3: Robotic Water Polo Platform GUI

In order to provide a fair competition experience for all competitors, the sensing (image capturing and processing), communications, and robotic fish subsystems cannot be modified. Competitors only modify the high-level strategies to implement features such as path planning, obstacle avoidance, high-level control algorithms, and game strategy. These user modified strategies are then utilized by the decision making subsystem. For interested readers, details on the robotic fish mechanical design, low-level modeling, and low-level control algorithms can be found in the references ${ }^{1,2,8}$. 


\section{Advanced Individual Studies Experience.}

The Advanced Individual Studies in Electrical Engineering (EE489) course has existed at USMA in some form or another for over three decades. Most offerings of the course are tailored specifically to the needs and qualifications of the individual student. EE489 normally requires a project with a significant undergraduate research component and submission of a final report. All of this work is completed under the guidance of one or more faculty advisors. EE489 project ideas can either be developed by the student who is proposing to take the course or by a faculty advisor. At other times, the course is used to pilot new electives with a small enrollment of 2-8 students. The course is a 3.0 credit hour course with a significant time commitment. Some of the projects in EE489 are continuations of prior student projects or are related to work done in one of the multidisciplinary design projects. In other cases, the student's work in EE489 can lay the groundwork for a more substantial multidisciplinary design project. Some examples of recent EE489 projects include Dense Wavelength Division Multiplexing using Erbium Doped Fiber Amplifiers, a Quadcopter Unmanned Aerial Vehicle (UAV) Communications Relay, and an unmanned ground vehicle (UGV) built on an electric go-cart platform. The quadcopter UAV communications relay project is an example of a former EE489 project that is now being pursued by a multidisciplinary team. The UGV project is an example of an EE489 project that has been continued on and off over the years by multiple students.

When creating an EE489 variation to support the Underwater Robot Competition we had to consider the resources available within our academic department (funding and lab space), the number of students both available and interested, and the timing of the competition. As we previously mentioned, the competition trip funding itself came from a source external to the department. In addition, all of the equipment and software were provided to us except for the computers used to execute the control software. We simply repurposed two older desktop computers that were on hand to solve this problem. Lab space was another challenge with the "fish tank" itself occupying a 3 meter by 2 meter space. Fortunately, one of the existing robotics labs was located on a ground floor and had both a raised floor area and a non-raised floor with built-in drainage and a utility sink. This allowed us to have both a "wet" and "dry" workspace for the project. While funding and lab space/environment did not end up being significant challenges for us, both should be taken into consideration by other institutions who wish to participate in the competition.

While the lab space problem was easily solved for us, it did pose one significant challenge that we had to overcome. The ceiling height was not large enough to use the lights provided with the platform. We initially attempted to use the overhead fluorescent lights in the room; however, we found that the reflected light from these caused some difficulties in the image capturing that must be noted. The robotic fish platform includes an adaptive underlay algorithm that attempts to address this issue ${ }^{2}$. Unfortunately, this algorithm didn't work well with the extremely bright lights and resulting large reflection. A workaround that we developed was to mount lights to 
each wall of the room and direct them such that the lights illuminated the playing field without a large surface reflection. This was a significant improvement, but was not ideal. Specifically, it has been difficult for the image capturing subsystem to follow movement in the corners of the pool, as our light settings create shadows in these areas.

When the project was proposed, student interest was high. Unfortunately, the academic department's enrollment of senior undergraduates who were eligible for an EE489 was low. The department decided that the project would be an individual project with only one student enrolled. However, the funding available for the trip to the competition itself was enough to support four students. The timing of the competition posed an additional problem, as it conflicted with final exams and other graduation requirements for our one student enrolled in the course. A partial solution to this problem was to request that the four students selected to attend the competition, who were juniors and sophomores without conflicts, assist and participate in an informal, non-graded capacity. This worked extremely well, as the students were extremely interested in both the project and the competition. This led to several of them giving up many hours of their free time in order to better prepare for the competition.

The platform discussed in Section 4 is relatively straight forward to set up. The software package comes with some pre-defined strategies to direct the robotic fish to swim from one point to another. The GUI interface makes it very easy for the students to utilize the image acquisition, image processing, and communication modules to move the robotic fish. It did not take long for our students to be able to navigate the robotic fish in the water, both through a manual control interface and using a pre-defined strategy. However the big challenge was how to make the robotic fish push the floating ball to a certain position effectively. This was essential in both obstacle avoidance situations and water polo games. In both cases, the robotic fish had to dribble the ball either to avoid obstacles or to score the goal.

It took quite a few weeks just for the students to get familiar with the behavior of the robotic fish and the ball under various commands. In working with the robotic fish, the students quickly discovered some of the challenges in underwater environments. For instance, it was evident that the dynamics of the robotic fish were unlike the ground vehicles they had seen in their earlier robotic classes ${ }^{5}$. While ground robots can exercise linear movement and instantaneous stoppage of motion, underwater robots cannot. Additionally, while ground robots have the advantage of significant friction forces, underwater systems do not. Whenever underwater robotic fish mechanical movement is stopped, the current forces consistently overwhelm the static forces attempting to stop the robotic fish. In fact, the robotic fish is almost never still, as water disruption always results in drifting of the robotic fish. This makes it very difficult for the robotic fish nose to be constantly pointing at the ball and hampered many attempts to use the robotic fish nose to push the ball forward as the robotic fish swims forward. Because of these 
uncertainties in the water, there is a large chance that the robotic fish misses the ball and it is almost impossible to obtain an accurate and consistent function for the robotic fish motion. This resulted in large test variability, even with identical variables and postures. For instance, it was not surprising for a robotic fish to dribble a ball for the length of the pool to take as short as 30 seconds or as long as 10 minutes $^{5}$. It seemed that much of this performance was based on chance, which highlights the innate difficulty and the frustration of underwater robotics.

As the project advisors, we had to make sure that our students made steady progress throughout the course. It was important that we gave students enough guidance so that they could focus on relatively simple, yet effective solutions. We asked our students to concentrate their effort on "push ball right" (assuming the goal is exactly to the right of the ball) only. This way the students could spend the majority of the time improving and testing one function instead of coding various scenarios without improving efficiency. Once the algorithm for "push ball right" was optimized, the same technique could be applied to "push ball left", "push ball up", "push ball down", and eventually "push ball at any angle" based on the positions of the goal and the ball. Moreover, the basic pushing function could be readily applied to multiple games with additional logic statements, such as the relay game and the water polo game. Overall, our students were able to complete all the strategies to attend the competition.

\section{Robotic Fish Class Schedule}

In this section we outline an example class schedule that we have created to support participation in the competition. This schedule has been modified and updated based on our experiences in the first year of conducting the class. The schedule is oriented around three blocks of material: Introduction, Basic Robotic Fish Control, and Design and Implementation of Game Strategies. Each block of instruction also includes recommended readings and references from a variety of sources. For completeness, full bibliographic information for all suggested readings is provided in the references section. While courses at USMA follow a 40-lesson schedule, we do not break this course into the typical schedule of one specific topic per lesson. The schedule is meant to be flexible, which we have found works well for the low enrollment of the course. Accordingly, the students and advisors can adjust the schedule based on the students' prior knowledge and pace of work. In addition, outside of fundamental electrical engineering courses the EE489 course has no official prerequisites. Therefore, we expect that students will enter the course with varied experience and specialties within the discipline.

Since the course is organized around the final goal of competing in the Robotic Water Polo Competition, official class meetings are typically conducted in the lab space. This allows students to immediately apply readings and discussions to the project. 


\begin{tabular}{|l|l|}
\hline \multicolumn{1}{|c|}{ Topic Introduction } & \multicolumn{1}{|c|}{ Reading/Reference } \\
\hline $\begin{array}{l}\text { Introduction to the Robotic Fish Platform and } \\
\text { Competition }\end{array}$ & [ROBO], [RULE] \\
\hline Robotic Fish Remote Control & \\
\hline \multicolumn{2}{|c|}{ Basic Robotic Fish Control } \\
\hline $\begin{array}{l}\text { Implementation and Testing of Example Robotic } \\
\text { Fish Strategies }\end{array}$ & [STRAT] \\
\hline $\begin{array}{l}\text { Implementation of Single Robotic Fish, Open-Loop } \\
\text { Ball Dribbling }\end{array}$ & [STROU], [KOEN] \\
\hline \multicolumn{2}{|c|}{ Design and Implementation of Game Strategies } \\
PID Control of a Single Robotic Fish & [NISE] ch 13, \\
\hline \multicolumn{2}{|c|}{ [DIGI] ch 9\&10 } \\
\hline Time-Based Competition Game Strategies & [PATH] \\
\hline Cooperative Control Strategies & [GAME1] ch 2-4, \\
\hline $\begin{array}{l}\text { Implementation of 1vs1 and 2vs2 Water Polo } \\
\text { Strategies }\end{array}$ & [GAME2] ch 1\&10 \\
\hline Final Research Paper &
\end{tabular}

Table 1: Example Course Schedule

\begin{tabular}{|c|c|}
\hline \multicolumn{2}{|l|}{ Readings/References } \\
\hline Long Title & [Short Title] \\
\hline $\begin{array}{l}\text { The Robotic Water Polo and Underwater Robot } \\
\text { Cooperation Involved in the Game }\end{array}$ & [ROBO] \\
\hline $\begin{array}{l}\text { Platform for Cooperation of Multiple Robotic Fish- } \\
\text { Robofish Water Polo }\end{array}$ & [COOP] \\
\hline $\begin{array}{l}\text { Rules of the International Underwater Robot } \\
\text { Competition }\end{array}$ & [RULE] \\
\hline $\begin{array}{l}\text { Performance Optimization and Coordinated } \\
\text { Control of Multiple Biomimetic Robotic Fish }\end{array}$ & [BIOM] \\
\hline $\begin{array}{l}\text { Path Planning for Robot Fish in Water-Polo Game: } \\
\text { Tangent Circle Method }\end{array}$ & [PATH] \\
\hline Competition Provided Example Strategies & [STRAT] \\
\hline The C++ Programming Language & [STROU] \\
\hline Accelerated C++ & [KOEN] \\
\hline Control Systems Engineering & [NISE] \\
\hline Programming Game AI by Example & [GAME1] \\
\hline AI Game Engine Programming & [GAME2] \\
\hline Microcontroller Based Applied Digital Control & [DIGI] \\
\hline
\end{tabular}


During the Introduction block, we begin the course by meeting with students in the lab space to familiarize them with the competition hardware, operating environment, and rules/objectives of the competition. We then allow them time to experiment with the robotic fish remote control interface that is included in the decision subsystem GUI. We find that this provides both an enjoyable experience for the students and an opportunity for them to witness the practical limitations of the robotic fish. As mentioned in Section 4, our students do not design or build the robotic fish. However, during the introduction we do discuss the design of the fish as understanding the design of the entire hardware system is crucial to completing the project.

Once we are satisfied that students understand the basics of the competition platform, we then transition to the Basic Robotic Fish Control block. We begin this portion of the course by having students explore and run the example strategies that are included with the decision subsystem. This allows them to begin understanding the Application Programming Interface (API) between the decision subsystem and the strategies. An understanding of the sensing information that is provided by the API (fish, ball, and obstacle coordinates; fish heading) and the allowable highlevel commands (fish speed, fish turning direction) must be obtained before moving on within this block. Once that is complete, we begin to have students implement their own algorithms for both open and closed-loop (PID) control of the robotic fish.

During the first iteration of the course, we found that our students spent much of their time focused on controlling one fish that was dribbling the ball. Therefore, it was not surprising that their strategies did not perform well during the 1vs1 and 2vs2 water polo competitions. Due to this, we expanded the Design and Implementation of Game Strategies block. We now include topics that focus on multi-agent control and sports game simulation theory. Finally, to meet requirements of the EE489 course, students are required to write an undergraduate research paper that is suitable for publication.

\section{Competition Results and Foreign Trip Experience.}

With this being our first year participating in the competition, our students' expectations were rather modest. Indeed, the team failed to make it out of the first round in both the 1vs1 and 2vs2 water polo games. However, to the delight and surprise of the team, they placed first in two of the time-based games. In hindsight, this isn't as surprising. As advisors, we had the students focus on the algorithms for moving the ball and let them mostly ignore the collaborative and game aspects of the competition. Since the time-based games mostly relied on efficient ball movement strategies, it makes sense that the team did well here. One of the other benefits of the competition was that it gave the advisors a lot of time to communicate and interact with the students. This gave us the opportunity to assess how well the competitive aspect of the project motivated the students. They all indicated that they would have been happy to simply "put up a good effort”, but were extremely pleased to win two events. This was especially evident when 
the team finished the "cycling with ball” event over one and a half minutes faster than any of the other teams that had already completed the event. The rest of the competitors began clapping and cheering for our team and congratulated them on their outstanding result.

This positive experience and friendly competitive atmosphere seemed to have the desired result as all four students expressed to us an increased interest in underwater robotics. Indeed, one was inspired by the free innovation league and on multiple occasions during the course of the competition sat down to brainstorm concepts. This eventually led to him proposing and completing his own EE489 project focused on adding true diving and surfacing ability to the basic robotic fish used in the global-vision league.

Another student enjoyed the competition and trip to China so much that he aggressively pursued the opportunity to spend a semester abroad in Nanjing. He pursued this opportunity even knowing that he would have to complete a significant amount of coursework in a short time prior to leaving and through distance learning. In addition, he was not only required to take Chinese language classes while abroad but also challenging engineering classes such as Dynamic Controls (admittedly, this course was offered in English).

Another aspect of the competition is the international nature and opportunity for new cultural experiences. Indeed, as we previously mentioned the funding source for this trip has a specific goal of increasing students' appreciation of diverse cultures. As part of the IAD, the students are required to fill out a short form discussing the experience, with a focus on the cultural aspects. Some quotes directly from these reports illustrate the students' experiences better than I could. For example, one student states that "Over the few days we were in Nanjing with these students we developed lasting relationships which we are continuing today.” She then goes on to say that “...our trip was full of fun and exciting explorations which will stay with me for my lifetime."6 Another student indicated that "Going on this IAD was an experience that I will ever forget."7.

\section{Conclusion and Future Work.}

In this paper we've concentrated on describing our experience with an international competition and some considerations in creating a class to support such a competition. Our experience with the Underwater Robot Open Competition and the excitement and inspiration that it generates in students has shown that it is well worth the extra effort and resources. While budget and lab space/environment concerns should be taken into consideration, these are not insurmountable hurdles. We plan to continue to offer the EE489 class to one student this year, with three to four other students participating in the trip and competition.

Future work will focus on two aspects. The technical aspect of improving performance in the competition is the first aspect. The competition results showed that the students' algorithms for 
moving the ball with the robotic fish nose are adequate, so little work will be done here. Instead, after familiarizing new students with the existing algorithms, we will have them focus on the cooperative nature of the problem and game strategies. In addition, during the last competition students noticed some opportunities for innovative strategies for interacting with the ball. We plan to have students explore these opportunities.

The second aspect of future work is on an administrative, or Electrical Engineering program, level. In order to capitalize on this success with a relatively small number of students, we need to ensure that the opportunity is known to other students. One possible method is to encourage former participants in the IAD to discuss their experience with other students. Another possibility is to actively advertise this unique opportunity when hosting department recruiting events or when discussing choice of majors with potential electrical engineering majors.

\section{References:}

[1] Z. Lee, G. Xie, D. Zhang, and J. Shao, “The Robotic Water Polo and Underwater Robot Cooperation Involved in the Game,” in Robotic Soccer, Ed. P. Lima, Vienna: Itech Education and Publishing, pp. 575-598, December 2007.

[2] J. Shao, and L. Wang, "Platform for Cooperation of Multiple Robotic Fish- Robofish Water Polo," in Proc. of the 46th IEEE Conference on Decision and Control, New Orleans, LA, Dec. 12-14, 2007.

[3] F. Runiu and C. Long. (2012, May 29). College of Engineering excels in 2012 Underwater Robot Competition. [Online]. Available: http://english.pku.edu.cn/News_Events/News/Global/9383.htm

[4] Rules of the International Underwater Robot Competition, (L. Ao, Trans) International Federation of Underwater Robot, 2012. [Online]. Available: http://robot.pku.edu.cn/

[5] G. Lu, “An Introduction to Robofish Water Polo and Marine Robotics”, unpublished.

[6] N. Wolf, "IAD Report”, unpublished.

[7] M. Abreu, "IAD Report”, unpublished.

[8] Y. Fang, J. Yu, R. Fan, L. Wang, G. Xie, "Performance Optimization and Coordinated Control of Multiple Biomimetic Robotic Fish,” Proc. of the 2005 IEEE International Conference on Robotics and Biomimetics (ROBIO), Hongkong and Macau, June 2005.

[9] Y. Jia, G. Xie, and L. Wang. "Path planning for robot fish in water-polo game: Tangent circle method." in Intelligent Control and Automation (WCICA), 2011 9th World Congress on, 2011, pp. 730-735.

[10] B. Stroustrup, The C++ Programming Language, $3^{\text {rd }}$ ed. Indianapolis, IN: Pearson Education, 2000.

[11] A. Koenig, B.E. Moo, Accelerated C++: Practical Programming by Example, Indianapolis, IN: Pearson Education, 2000.

[12] N.S. Nise, "Digital Control Systems," in Control Systems Engineering $6^{\text {th }}$ ed. Hoboken, NJ: Wiley, 2011 , pp. 723-780.

[13] D. Ibrahim, Microcontroller Based Applied Digital Control, Hoboken, NJ: Wiley, 2006.

[14] M. Buckland, Programming Game AI by Example, Sudbury, MA: Jones \& Bartlett, 2005.

[15] B. Schwab, AI Game Engine Programming $2^{\text {nd }}$ ed., Boston, MA: Course Technology, 2009. 